\title{
State of the Art of Multi-Dimensional Modeling of Engine Reacting Flows
}

\author{
A. D. Gosman ${ }^{1}$ \\ 1 Imperial College of Science, Technology \& Medicine, Exhibition Road, SW7 BX London and \\ Computational Dynamics Ltd, 317 Latimer Road, London W10 GRA - United Kingdom \\ email:d.gosman@ic.ac.uk
}

\begin{abstract}
Résumé - État de l'art de la modélisation multidimensionnelle pour les écoulements réactifs dans les moteurs - Cet article dresse un aperçu de l'état de l'art et des perspectives de l'utilisation de la modélisation 3D pour l'analyse et le développement des moteurs. Les aspects abordés comprennent l'acquisition des géométries, la génération des maillages, les méthodologies numériques, la modélisation mathématique des phénomènes physiques importants, l'exploitation des calculateurs parallèles et l'application industrielle. Les conclusions principales de cette analyse font apparaitre les points suivants :

- les méthodologies numériques et la génération des maillages, associées au calcul parallèle, permettent maintenant de raccourcir notablement le cycle total de simulation;

- la modélisation physique a atteint sa maturité dans la plupart des aspects relatifs à la simulation de la combustion en allumage commandé ; en revanche, elle reste encore à acquérir en ce qui concerne la combustion Diesel et certains aspects des processus reliés aux sprays ;

- globalement, l'utilisation de la simulation 3D pour la conception des moteurs est en forte augmentation ;

- les développements futurs, dont l'utilisation de la LES, promettent une amélioration croissante des résultats.

Mots-clés : moteur, combustion, simulation 3D, aperçu.
\end{abstract}

\begin{abstract}
State of the Art of Multi-Dimensional Modeling of Engine Reacting Flows - An overview is provided of the state of art and future prospects for the use of CFD simulation in engine analysis and design. The aspects covered include geometry handling and mesh generation, numerical solvers, mathematical modeling of key physical phenomena, exploitation of parallel computers and industrial application. The main conclusions are that:

- solver and mesh generation methodology developments, allied to parallel computing technology, now enable much more rapid turnaround in CFD simulations than hitherto;

- physics modeling is at an advanced stage for most aspects of SI combustion simulation, but less so for Diesel combustion and for spray-related processes generally;

- the overall picture is one of rapidly-increasing use of CFD for engine design;

- future developments, including the use of LES, promise even better results.

Keywords: engine, combustion, CFD, review.
\end{abstract}

\section{INTRODUCTION}

\section{The Challenges}

The development of CFD methodology and computer codes for engine simulation is a challenging task, for a number of reasons. First and foremost, the reciprocating engine exhibits many complex features and phenomena, perhaps more than any other widely-used mechanical device. As a starting point, there is geometrical complexity, because the shapes of the combustion chamber, piston crown and inlet and exhaust ports are key features in the design, along with the valve motion. Then there are the many simultaneous interacting thermofluids processes, including:

- non-stationary turbulent flow, heat and mass transfer;

- injection, atomisation, disperson and evaporation of liquid fuel;

- ignition and combustion of the gaseous fuel;

- the consequent formation of harmful pollutants, including oxides of nitrogen, unburnt hydrocarbons and particulates. 
These features place stringent demands on the two core areas of CFD, namely mathematical modeling and numerical solution methodology.

On the modeling front, the task is to assemble a sufficiently complete, accurate and economical mathematical description of the aforementioned physical process. "Sufficiency" in this context means that all the essential features are represented in the model equations, to an accuracy which is adequate for the designer's purposes and at an acceptable cost of solution. As is well known, to meet these requirements it is necessary to introduce approximations, such as the Reynolds Averaging (RA) approach to turbulence and reduction of complex chemical kinetics mechanisms to simpler forms.

The task for the numerical analyst is to assemble an algorithm for solving the model equations in a flexible, accurate, economical and speedy fashion. The flexibility requirement refers both to the range and form of model equations and to the engine geometry, whose complexities were mentioned earlier. Since most numerical algorithms employ some form of computing mesh, it is primarily this which determines the geometry-handling capabilities. The remaining numerical requirements are collectively determined by the discretisation accuracy, solver efficiency and computer implementation. The last-named has become particularly important in recent years due to the improved availability and usability of parallel computers.

Substantial advances have been made in all the aforementioned areas, particularly during the past decade, such that CFD simulation now has reasonable credibility within the engine community. However there are additional requirements to be met for it to become a design tool: they are adequate ease of use and rapid overall turnaround, starting from a CAD description of the geometry and other input data and ending with the simulation results, in a digestible form. The necessary elements to achieve this comprise rapid mesh generation (and control during the analysis to accommodate the piston and valve motions), sufficiently small CFD simulation time and appropriate data visualisation/analysis tools.

Finally, a perhaps less obvious challenge to CFD simulation is the limited availability of suitable experimental data to guide the development and assess the accuracy of the methodology. Benchmark engine experiments are notoriously difficult to perform: many different quantities need to be measured to properly characterise the operating conditions (e.g. time-varying fuel and air flow rates, pressures and temperatures; wall temperatures). Furthermore assessment data other than the usual cylinder pressure and exhaust composition measurements require access, optical or otherwise to the combustion chamber which is difficult in itself and can introduce further complications, such as flow disturbance and unquantified additional leakage.

\section{Purpose and Contents}

This paper provides an overview of the state of art in the numerics and modeling areas mentioned in the previous section and thus of the overall status of CFD engine simulation as a research and design tool. Because the scope is wide, the depth is necessarily limited, but many of the topics are more extensively covered in other papers in this conference, which will be referred to along with other supporting references.

The review commences in the next section with the solution methodology, encompassing numerical algorithms, mesh structure and generation, postprocessing and implementation on parallel computers. Following this in Section 2 the physics modeling is surveyed, including turbulence, sprays, combustion and emissions. A few example applications are included in this part, indicating the overall accuracy achievable.

\section{SOLUTION METHODOLOGY}

All of the widely-used codes for engine simulation employ finite-volume methodology (FVM), so this review will focus on this approach. However alternative types of method are being used or developed, two of which are described in other papers in this conference $[1,2]$.

\subsection{Flexible Meshes}

One of the key developments of FVM in recent times has been recognition that it can be made far more flexible in respect of mesh structure, and hence of geometry-handling capability, than was originally supposed. A step in this direction was to introduce multi-block hexahedral structures, as employed in the IFP KIVA-MB [3], KIVA-III and [4] FIRE $^{1}$ codes, which facilitate fitting of ports and cylinder head and piston recesses, along with other benefits. The Speed [5] code carries this a stage further by allowing general unstructured hexahedral meshes. Even greater flexibility is available in the STAR-CD ${ }^{2}$ and SPEED-STAR codes, including the options to:

- work with non-hexahedral meshes, comprised of tetrahedra, triangular prisms or even arbitrary polyhedra and also mixtures thereof ("hybrid meshes");

- locally subdivide existing cells to form regions of embedded refinement;

- introduce internal sliding interfaces, allowing relative motion of adjacent mesh zones along them;

- attach zones of dissimilar mesh structure without requiring continuity between them "arbitrary interfacing".

1 FIRE is the trademark of AVL List GmbH.

2 STAR-CD and SPEED-STAR are trademarks of Computational Dynamics Ltd. 
Further information about the above capabilities, which enable virtually any engine configuration to be meshed and also open the door to the use of a range of automatic mesh generation techniques, can be found in [7]. Many of them have been used in the engine example of Figure 1, showing a Star-CD mesh for a DI-SI engine chamber. It consists of a hybrid assembly of tetrahedra and triangular prisms to facilitate geometry-fitting, mesh motion and layer addition and removal. Sliding interfaces are used to accommodate the valve movements and arbitrary interfacing is employed to attach the piston bowl mesh to that for the swept volume, so that alternative piston designs can easily be introduced.

Hybrid meshing is also allowed in the recently-developed CHAD code [6].

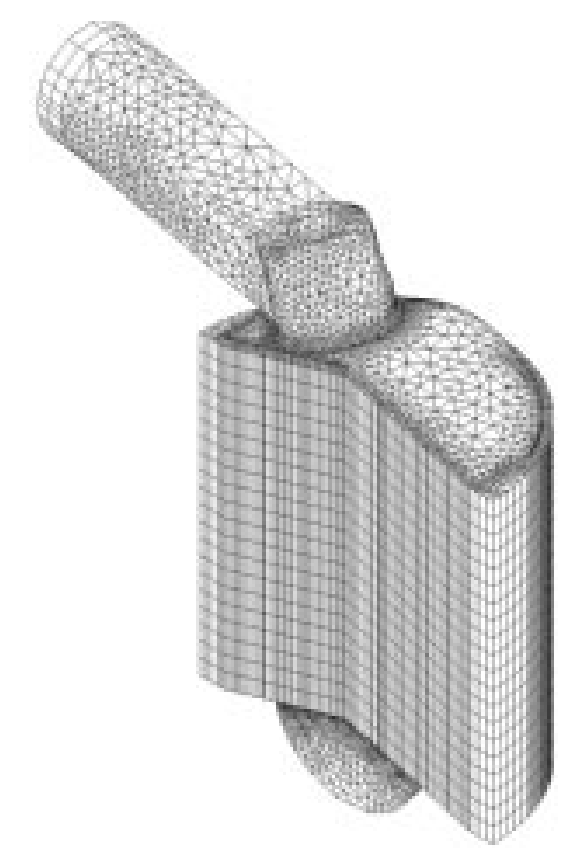

Figure 1

Unstructured hybrid computing mesh for DI-SI engine simulation, produced using rapid meshing techniques.

\subsection{Accurate Discretisation}

Improvements in accuracy have been achieved by the introduction of "smart" higher-order (usually second-order) spatial discretisation schemes (e.g. $[6,8]$ ) which combine better resolution with the ability to avoid the numerical wiggles of traditional higher-order discretisation. Similar developments are now being made to temporal discretisation. Despite these advances, adequate resolution of the complexities of engine flow and combustion still requires relatively large meshes - of around 500000 cells - with associated big computing overheads. In practice however to reduce the latter coarser meshes have normally been used, resulting in numerical discretisation errors, usually unquantified and therefore indistinguishable from those from other sources. Fortunately, thanks to computer-related developments described below, this situation is now changing.

\subsection{Implicit Solution Algorithms}

Another important trend in engine CFD has been the move towards fully-implicit solution algorithms (e.g. [5, 6, 9, 10]), because of the freedom they offer from mesh-related stability restrictions on time step size. This, along with the availability of efficient linear equation solvers, has produced substantial computing time (and hence cost) savings.

\subsection{Parallel Computing}

The past five years has seen a sea change in the availability of high-capacity computing hardware at relatively low cost (compared with traditional vector supercomputers), thanks to the development of the new generation of parallel machines. These rely on "commodity" workstation RISC processors, linked by high-speed networks, controlled by Unix operating systems and programmed with standard languages. This combination enables reductions in computing time which, with suitable codes, can be proportional to the number of processors. The latter can be in the tens, hundreds or even thousands, according to the type of machine. Such speedups are made possible by the use of a combination of "domain decomposition" whereby the mesh is partitioned across the available processors in blocks of equal numbers of cells and "message passing", in which the necessary data transfers are made between the processors to keep them working in synchronisation.

In order to exploit these machines it is necessary to appropriately structure the numerical algorithms and computer codes. That this is possible with current FVMbased engine codes is demonstrated in Figures 2-5, taken from a recent study partially reported in [11]. Figure 2 shows the Mercedes-Benz M111 four-valve pent-roof SI engine and Figure 3 displays a mesh of some 300000 cells fitted to this (the symmetry of the engine is exploited, so the effective fullengine mesh actually has 600000 cells) for the purposes of simulation. The nature of the partitioning used for parallel calculations is indicated by the different colours: in this example it is for 8 processors.

Figure 4 shows how the total computing time for a fixed simulation period varies with the number of processors and illustrates quite clearly the substantial reductions which can be achieved. In practice, this means that by using sufficient processors a case of this size can be completed in one or two days. This, along with other developments mentioned below, enables for the first time engine simulations to fit within the design cycle. 


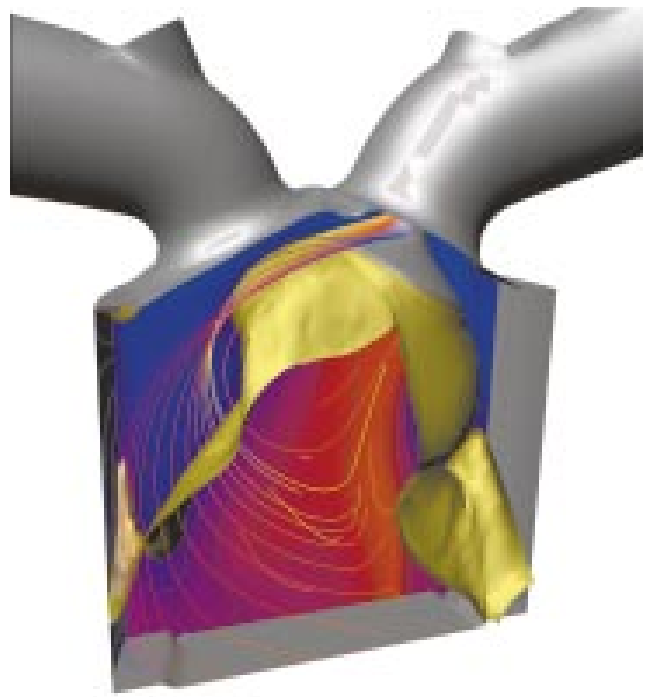

Figure 2

Geometry of Mercedes-Benz M111 four-valve SI engine, with flow visualisation.

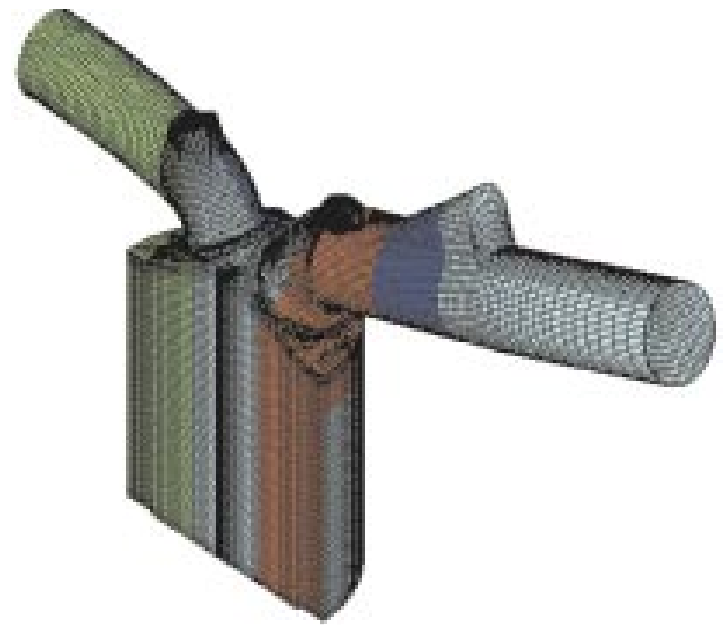

Figure 3

Computing mesh for M111 engine, comprising 300000 cells in half-section. Colouring indicates mesh decomposition for parallel calculation on eight processors.

\subsection{Automated Mesh Generation}

Another obstacle to the use of CFD in engine design has been the mesh generation process. In the past this has been difficult and time consuming, because the available manual mesh generation techniques required considerable expertise and effort to address the complex shapes and moving parts of the combustion chamber and intake and exhaust systems. This is because the geometries have had to be hand-fitted,

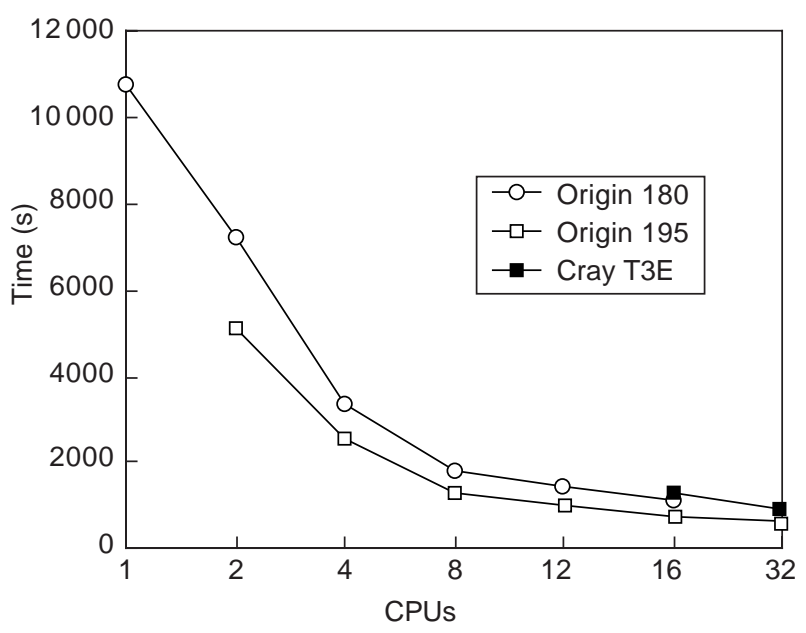

Figure 4

Data on computing time for M111 simulation on parallel machines as function of number of processors used.

while working within fairly severe constraints on mesh structure; and then the mesh has had to be adjusted, again manually, to accommodate the moving parts, while ensuring that it does not become excessively distorted. The latter requirement usually entails local alterations to mesh topology, such as addition or removal of portions in the valve gaps, during the engine cycle.

Recently automated techniques have been developed, thanks in part to the greater mesh flexibility described earlier, which have dramatically reduced the meshing times. One such approach [12] was used to generate the mesh shown in Figure 1. It operates in two stages: firstly the ports and cylinder head and piston surfaces are meshed (in this example by an automatic tetrahedral generator); and then the in-cylinder volume mesh is automatically produced, including the dynamic changes required for the piston and valve movements.

Another method, described in [11] and illustrated in Figure 5, uses the notion of generic templates, whose overall topology and built-in dynamic changes instructions are designed once for all for particular families of engines. Facilities are then provided to allow the template mesh to be "moulded" to fit specific engines within the family. Figure 5 shows the template used to produce the mesh for the Mercedes-Benz M111 engine: it has a multi-block hexahedral structure, although there are isolated departures from this, not very visible, to cater for particular geometrical details. Also displayed is the CAD surface presciption for engine and the final working mesh fitted to the CAD geometry by projecting the template mesh surfaces onto it and then adjusting the interior vertices.

Yet another automated meshing approach is described elsewhere in these proceedings [13]. 

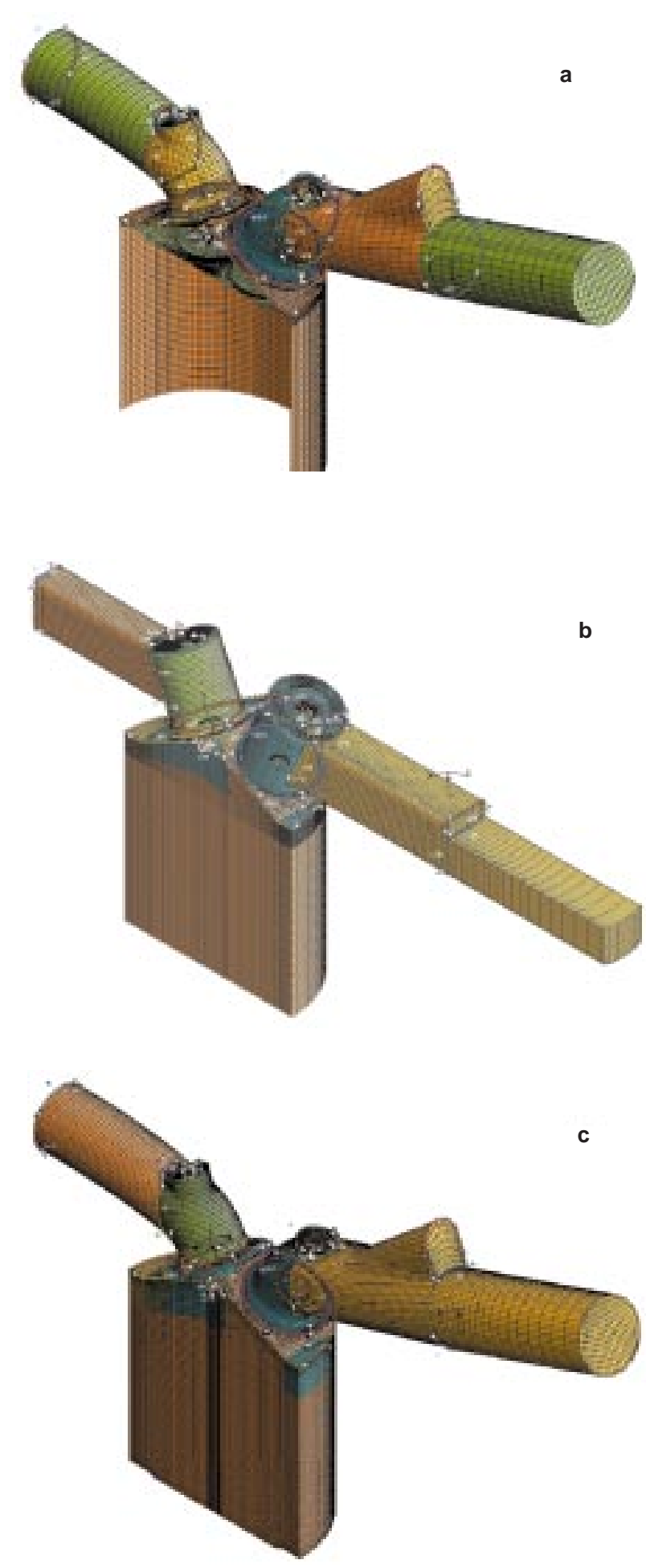

Figure 5

Illustration of template-based mesh generation for M111 engine:

a CAD model of engine;

b template mesh;

c final mesh after projection onto CAD surfaces.

\subsection{Improved Post Processing}

The huge volume of data produced by a 3D engine simulation has posed problems of storage and interpretation from the early days of engine CFD. Data storage devices have become larger and cheaper, although this has been offset to some extent by the use of larger meshes. It seems however that the development of rapid visualisation software tools [14], some of which are able to run on parallel machines, will increasingly enable results to be visualised as they are being generated, which offers a number of advantages. Figure 2 shows one form of visualisation generated by such a tool [11]. Advances are also being made in the modes of visualisation available to the design engineer: animation of results is becoming relatively straightforward and visualisation using virtual reality techniques and equipment is now possible.

\section{PHYSICS MODELING}

The modeling of the physical processes in engines, of which turbulence and its effects on heat and mass transfer and chemical reaction are the most paramount, continues to be done primarily within the Reynolds Averaging (RA) framework: indeed this is exclusively the case in industrial use. As will be discussed later, the alternative Large Eddy Simulation (LES) approach is likely to be an attractive alternative to RA in the not too distant future.

Whatever approach is used, the accuracy of a CFD simulation will be determined by the adequacy of physics models if and only if the mesh and time intervals are small enough to reduce discretisation errors to acceptable levels. This fact merits emphasis because for reasons explained earlier in many instances judgements about adequacy of models have been made on the basis of results corrupted to some extent by resolution errors. Similar comments apply to coupled physical phenomena: for example, the accuracy of a spray calculation will clearly be compromised by errors in the turbulence modeling; and a combustion calculation will feel the cumulative effects of both the turbulence and spray modeling errors.

\subsection{Turbulence Modeling}

Most engine codes employ some version of the well-known $\mathrm{k}-\varepsilon$ model and associated wall functions for the calculation of the RA turbulent Reynolds stresses, scalar fluxes and characteristic turbulence time and length scales required for engine simulations. The default choice tends to be the original Launder-Spalding version [15], with some modifications for compressibility effects $[16,17]$. Some more recent variants (e.g. $[18,19])$ are claimed to better represent effects such as turbulence anisotropy, but they have not yet been properly assessed in the engine context.

Other turbulence representations have been explored, including complex Reynolds stress transport models [20, 21], 
but lack of widespread use suggests that the benefits, if any, are marginal. Given that a similar picture exists in other areas of CFD application, this is scarcely surprising.

The accuracy of flow prediction using the standard k- $\varepsilon$ model is variable. Generally speaking it is poorest during the induction stroke, especially with regard to the turbulence intensity, which is usually substantially underpredicted [21, $22]$. Better and often satisfactory agreement is however obtained later in the engine cycle (e.g. [23]), due probably to the facts that the induction-generated turbulence rapidly decays and is replaced by that produced by the prevailing mean velocity gradients; and the large-scale flow structure becomes simpler. It should be noted that the turbulence parameters $\mathrm{k}$ and $\varepsilon$ are particularly sensitive to mesh resolution, tending to be underestimated if the mesh is too coarse. Fortunately the turbulence intensity $u^{\prime}(\propto \sqrt{k})$ and integral length scale $\ell\left(\propto \mathrm{k}^{3 / 2} / \varepsilon\right)$, which determine important quantities like turbulent diffusion coefficients $\left(\propto u^{\prime} \ell\right)$ and turbulent flame speeds $\left(\sim \propto u^{\prime}\right)$, are less mesh-sensitive.

\subsection{Heat Transfer and Mixing}

Heat transfer predictions obtained with the standard k- $\varepsilon$ model and wall functions are commonly believed to be underestimates. This has been attributed to weaknesses in the wall functions and several attempts have been made to address these (e.g. [24]). However work in this area has been hampered by the fact that there are apparently remarkably few published well-characterised and detailed engine heat transfer experiments on which to base judgements and developments.

Useful predictions of gaseous fuel-air mixing (mixture preparation) can be obtained with the standard model. This has demonstrated for example in [25], where it has been possible to characterise and rank different MPI SI engine intake arrangements in terms of the mixture stratification pattern they produce around the time of ignition. Similar studies are reported in [26, 27] for DI-SI engines.

\subsection{Fuel Injection and Sprays}

The modeling of fuel injection processes is an essential part of engine simulation. This has always been the case for Diesels and has come to be increasingly so for SI engines as manufacturers haved moved to port-injection and now directinjection operation.

As reviewed elsewhere in these proceedings [28] and in [29], models of different levels of capability are now available for nearly all fuel injection processes (at least for Diesel engines), including:

- pump/line simulation;

- flow in the nozzle including cavitation [28, 30, 31]; atomisation [28, 32-34];

- spray motion and evaporation [29, 35];

- wall impingement [36,37] and film formation and evaporation [38-40].
In general however it has proven to be necessary to empirically "tune" coefficients or other inputs to the models by reference to experimental data to obtain satisfactory quantitative predictions, particularly when dealing with new designs or substantially different operating conditions. This is undoubtedly due in part to weaknesses in some of the simulation components, with cavitation and atomisation modeling being the prime suspects. This applies to all injector types, but particularly to those for SI engines (both port and direct injection), for which the modeling is less advanced. It is also becoming widely recognised that the (nearly) universal practice of calculating the droplet motion by the stochastic Lagrangian method may result in irreducible discretisation errors, at least for narrow-angle sprays, for which this approach is not really suitable.

It should also be noted that although judgements of spray modeling accuracy are usually made by reference to quantities such as penetration and (less frequently) droplet sizes and velocities, the real quantities of interest are the distributions of mixture concentration and temperature, because these are the main determining factors on ignition and combustion: however they are difficult to measure, especially within the spray. Notwithstanding all these caveats, it should be stressed that there is ample evidence that the present spray modeling framework is already capable of producing useful results, when used with due care.

\subsection{SI Combustion and Emissions}

Over the past decade substantial advances have been made in the modeling of combustion in SI engines. Initially the emphasis was on homogeneous-charge operation, but latterly attention has moved to stratified-charge combustion, motivated particularly by the strong interest in DI-SI engines.

The progress can be attributed to the development of a new generation of models which explicitly take into account the fact that SI engine combustion largely occurs in the wrinkled flame regime [41]. Examples are the "CFMITNFS" development of the Coherent Flame Model by Poinsot, Boudier, Baritaud and coworkers [42], the Weller "Wrinkled Flame Model" [43, 44] and the "G-Equation Model" proposed by Peters [45] and others. While these differ in important respects, the common characteristics are that the instantaneous flame is regarded as a thin surface which propogates normal to itself at the local laminar flame speed and is wrinkled and strained by the turbulent flow field. Separate submodels are developed for the ensembleaverage flame wrinkling (leading to the local flame area); and the flame propagation speed. The local turbulent burning rate is proportional to the product of these quantities. One of the advantages of this approach is that the underlying assumptions are directly verifiable, either by experiment or DNS (e.g. [46]), in contrast to the "fuzzy physics" of the earlier and more empirical eddy break-up models. 


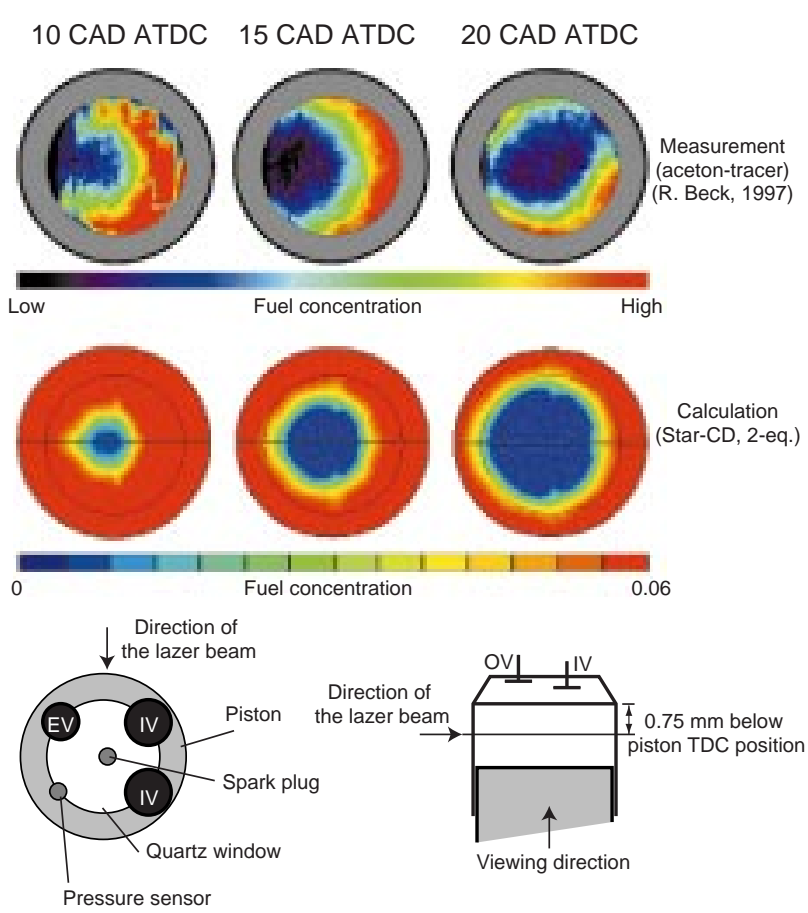

Figure 6

Comparison between measured and predicted (using Weller wrinkled flame model) flame propagation in M111 engine, running in $\mathrm{HC}$ mode.

M111, homogeneous combustion

Full CAD, $1500 \mathrm{tr} / \mathrm{min}$, ignition timing $=10^{\circ} \mathrm{CAD} \mathrm{BTDC}$,

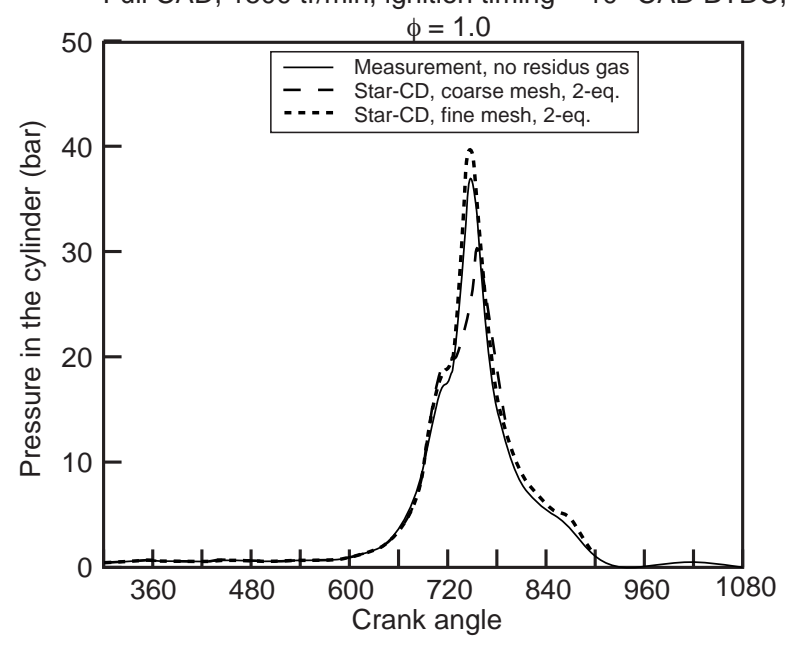

Figure 7

Comparison between measured and predicted cylinder pressures for M111 engine.
There is ample evidence that these new models can produce useful predictions of engine combustion in the homogeneous mode (e.g. [43, 44, 47]). By way of example, Figures 6 and 7 show comparisons between measured and predicted flame progress and cylinder pressure respectively, taken from [11] and pertaining to the Mercedes-Benz M111 four-valve engine illustrated earlier in Figure 2. The calculations were performed on a parallel machine using a range of different computing mesh densities, the finest of which is illustrated in Figure 3. The agreement between the measured and predicted flame propagation for the finest mesh is within experimental uncertainty; and the accuracy of pressure prediction is also satisfactory.

In recent times all of the wrinkled flame models mentioned above have been extended to the partiallypremixed regime [48-50], to make them applicable to stratified-charge engines, including the DI-SI type. This has been done by determining the local stoichiometry from the mixture fraction field, on the assumption that it is effectively homogeneous at the small scales.

DI-SI engine combustion studies with some of these models are presented elsewhere in these proceedings [13,50] and show encouraging agreement with measurements, especially when the difficulties of modeling the spray are taken into account (in this regard, it is usually necessary to resort to measured spray input conditions for DI-SI injectors).

Figure 8 shows an extract from a more fundamental study [49], in which the partially-premixed Weller model is used to compute stratified combustion at low turbulence levels in a constant-volume pressure chamber, previously investigated experimentally by Moriyoshi [51]. As indicated, the prediction of the pressure rise for an initially vertically stepstratified mixture with equivalance ratios of 1.0 and 0.4 in the lower and upper regions respectively is within the scatter of the individual repeat experiments. The predicted flame structure, also displayed, exhibits the same qualitative features evident in the photographs in [51].

Concerning $\mathrm{NO}_{x}$ emissions, adequate predictions of trends, and sometimes absolute levels, have been obtained for $\mathrm{HC}$ engines by some workers using standard extended Zeldovich chemistry, equilibrium burnt-gas thermochemistry and ensemble-averaged temperatures and concentrations, at least for lean mixtures. However it is generally acknowledged that for greater accuracy and a wider range of equivalence ratio the following are required:

- a more general burnt gas chemistry model allowing for phenomena such as non-equilibrium (particularly of $\mathrm{CO}$ );

- modeling of turbulence-chemistry interactions, especially due to temperature fluctuations;

- use of conditional analysis to obtain better predictions of burnt (and unburnt) gas temperatures and concentrations within the flame brush. (Similar comments apply to the analysis of knock in the end gases). Some progress has been made in these areas $[25,52]$. 

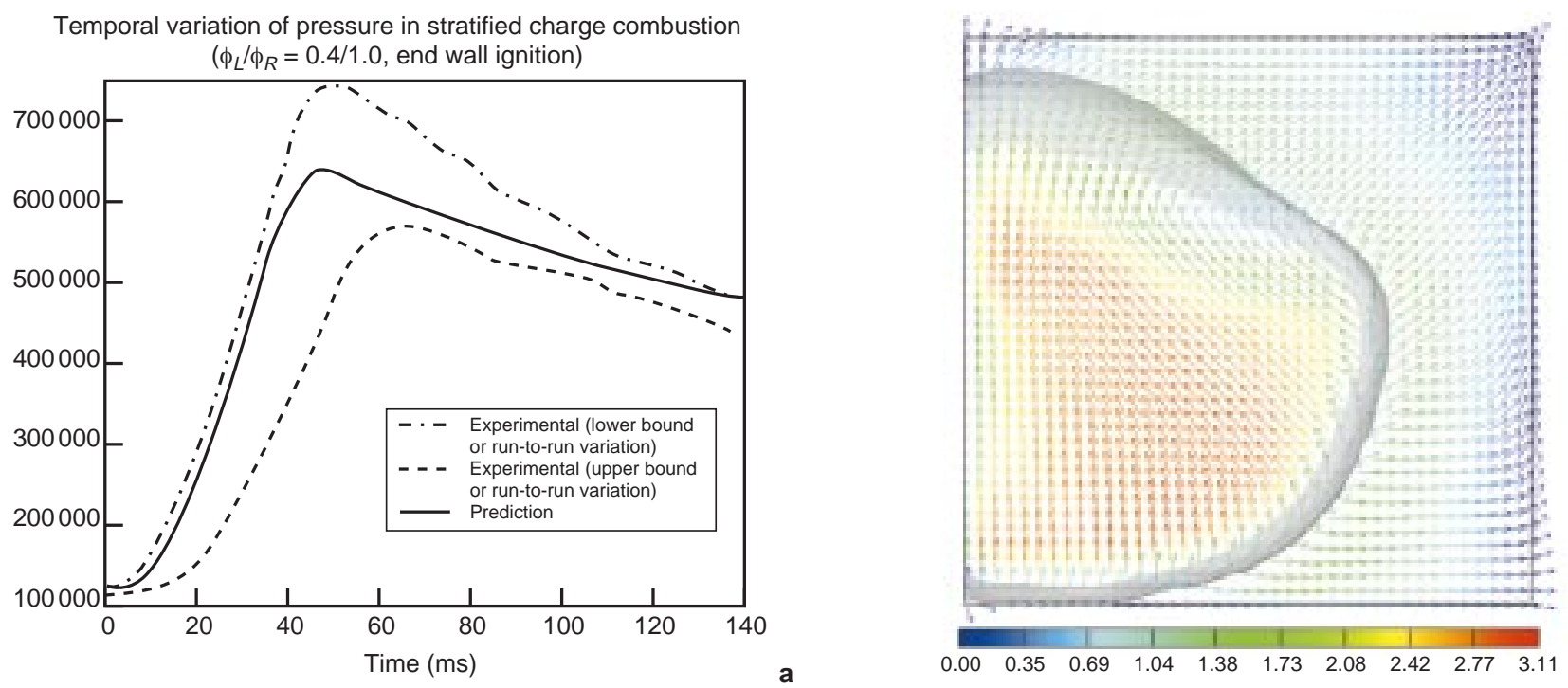

Figure 8

Calculation of partially-premixed combustion in pressure chamber using Weller model:

a comparison between measured and predicted pressure variations;

b predicted flame structure at around mid-burn, showing effects of stratification).

Methods for predicting unburned hydrocarbons (UHC) are less advanced. In $\mathrm{HC}$ engines these are generally regarded as originating from trapping of fresh charge in ring and other crevices during compression and release and incomplete oxidation during expansion [53]. There are smaller contributions from fuel dissolution in the oil film. The entrapment and release processes can in principle be simulated, but adequate low-temperature oxidation chemistry models are not yet available. DI-SI and other modes of stratified charge operation introduce additional sources of UHC, including bulk quench (inevitable if the local mixture strength falls outside the flammability limits) and poor mixing due to bad spray characteristics, which can also promote increased UHC via fuel deposition on walls. Here too more model development is needed.

\subsection{Diesel Combustion and Emissions}

The modeling of Diesel combustion is at a less advanced state than that for SI engines. Arguably, the main reason is that it is more difficult in a Diesel simulation to isolate combustion-related errors from those arising from the numerics and the flow and spray physics modeling. This is so because in the Diesel:

- ignition occurs naturally, rather than being imposed by a spark;

- the coupling between the fuel injection and ignition/ combustion processes is particularly strong, because they occur simultaneously;

- the presence of the spray inhibits obtaining detailed measurements to guide combustion model development.
The most popular Diesel modeling approach is to employ a simplified laminar kinetics (SLK) model for the ignition process, in which a simplified reaction scheme is used and turbulence-chemistry interactions are largely ignored; and then switch to an eddy breakup (EBU), model for combustion. This will hereafter be referred to as the SLK/EBU approach. There are many variants, according to the choices of SLK representation, transition criterion (typically the level or rate of change of temperature) between ignition and combustion and form of EBU, along with other details. A model of this kind, incorporating a variant of the well-known Magnussen [54] EBU has been developed at the University of Wisconsin by Reitz and coworkers [55] and successfully used by them to predict combustion in large truck engines [56], apparently with limited need to "tune" model coefficients. However experience of use of this and other SLK/EBU variants for smaller engines by various workers (e.g. [57, 58]) has generally been that although reasonable agreement can also be obtained for these, it is often necessary to adjust one or more model coefficients for each operating condition.

Within the SLK/EBU framework, soot emissions are usually calculated with a phenomonological model such as that of Hiroyasu [53] and $\mathrm{NO}_{\mathrm{x}}$ with the standard extended Zeldovich model [53]. Patterson et al. [56] found that this approach gave adequate predictions of trends with changes in operating conditions after adjustment to give agreement at one condition. Clearly this is only likely to be true if the combustion trends are being correctly predicted.

Other Diesel combustion modeling frameworks have been proposed, including ones based on flame area [59] and laminar flamelet [60] concepts. Thus far limited success has 
been achieved with the former, although the fact that they work well for SI combustion suggests that they are worth pursuing. The most recent model within the latter category is "RIF" (standing for "Reactive Interacting Flamelet", developed by Peters and colleagues, and described elsewhere in these proceedings [60]. The essential features are:

- a "full" all-embracing chemistry description is used for ignition, combustion and emissions (including both soot and $\mathrm{NO}_{\mathrm{x}}$ );

- all of these processes are assumed to occur in the (unsteady) flamelet regime, with presumed-pdf modeling of turbulent interactions;

- the transient flamelet equations are solved in a separate RIF code simultaneously with the CFD code, with continuous interchange of pressure, temperature, concentraton and other information between the two codes.

The computing requirements of RIF are too large to allow it to be applied at every CFD mesh point (the maximum reported number of simultaneous RIF calculations is around 20, many orders of magnitude less than the mesh size) so spatial averaging of the CFD results is required, along with some key assumptions about the controlling factors in the combustion processes. Thus, in essence RIF offers detailed flamelet modeling of the chemistry and its interaction with the turbulent flow field at the price of (subsantially) reduced spatial resolution. Although this compromise somewhat goes against the grain of eliminating resolution errors, the published applications suggest that RIF merits further consideration as an alternative to the SLK/EBU and other approaches.

\subsection{Future Developments}

There is clearly scope for further development of the RA approach, in areas already mentioned; and there are grounds for optimism that further improvements in accuracy will result. In the meantime however the alternative LES framework has also been developed and assessed for engine simulation, with promising results $[22,61]$.

LES has two main attractions over RA modeling for engines: firstly, it offers the possibility of individual cycle simulation and thus variability between cycles; and secondly, it is potentially more accurate, since the need for turbulence modeling is confined to the subgrid scales. Also, the fact that LES requires 3D unsteady calculations is not a drawback as it is for stationary flows, since the same requirement applies for RA engine calculations. However there are counter arguments, namely: additional cycles imply a proportional increase in the computing overheads; much of the important spray and combustion physics occurs at subgrid scales and therefore offers essentially similar challenges as in RA modeling; and boundary condition treatment for LES is relatively less developed, especially economical methods for industrial applications.
Indications that the benefits of LES may already outweigh the disadvantages can be found in the references cited above, which include the paper by Howarth [22] in the present proceedings. This study clearly demonstrates that LES is able to produce superior predictions of induction-generated flow that any of the current RANS approaches, including full Reynolds stress transport models. It also demonstrates that the large apparent turbulence intensities observed during this stage are due to variations in the large eddy behaviour, a feature of turbulent flows which RA modeling does not capture. This could also explain why RA calculations sometimes underestimate the degree of mixing between the fresh charge and residual gases.

Another demonstration of current LES capabilities is provided in Figure 9, taken from current research at Imperial College and showing LES simulations of the growth of a premixed turbulent flame initiated from a spark in a homogeneous turbulent field. The experimental data were obtained in a fan-stirred bomb by Sheppherd and coworkers [62] and the simulations were performed by the LES version of the Weller wrinkled flame model, described in [63].

Both the simulations and the experiments show variability between individual realisations, which can clearly be attributed to the stochastic nature of the turbulent flow field, leading to different conditions at the time of spark for each realisation. Similar effects are seen in engines. The fact that greater variability occurs in the measurements than predictions in this case is probably due to the fact that the numerical ignition procedure employed is "too reliable", i.e. it does not replicate the initial variability in the real ignition process clearly visible in the experimental results.

Iso-octane air: $\mathrm{phi}=1.0, \mathrm{Ti}=358 \mathrm{~K}, \mathrm{Pi}=1 \mathrm{bar}, 2000 \mathrm{tr} / \mathrm{min}$

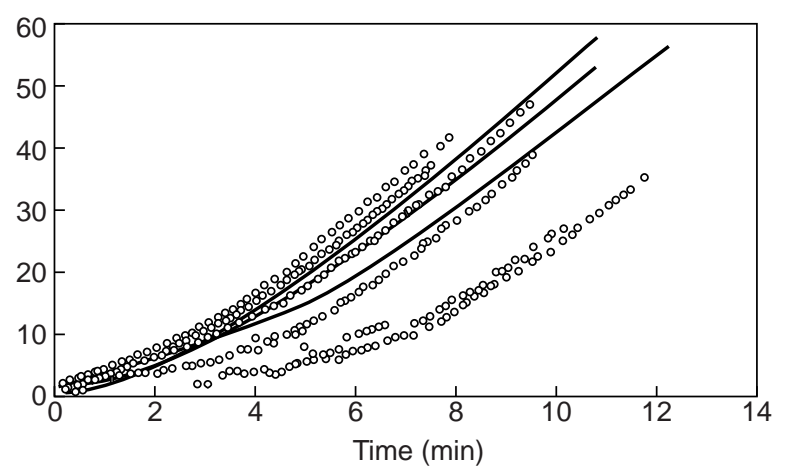

Figure 9

LES simulations (curves) of premixed combustion in homogeneous turbulence and comparison with experiment (points) - different curves and points refer to individual realisations. 


\section{CONCLUSIONS}

On the basis of the developments described in this overview it is reasonable to claim that CFD engine simulation is now at the stage where it is useful for engine design, as opposed its earlier mainly research applications. This enhanced utility is due primarily to substantial improvements in capabilities, speed of turnaround and accuracy. The key enabling developments have been more flexibile geometry handling, automated mesh generation, faster and more accurate numerical solvers and improved and extended physics models, allied to commercial code development. Speed and accuracy have also been enhanced through the ability to exploit the new generation of parallel computers, enabling both shorter run times and better mesh resolution.

In the future, further improvements can be expected in every aspect of engine simulation covered in this survey. They are particularly needed in certain current areas of weakness, notably the modeling of spray atomisation and Diesel combustion and emissions. Although there is still substantial untapped potential in the Reynolds-averaging modeling framework, LES will undoubtedly emerge as a complementary approach, able to provide additional and sometimes more accurate information, particularly in regard to the nature and origins of cycle-to-cycle variations.

\section{REFERENCES}

1 Sainsaulieu, L., Buchou, C., and Maries, C. (1999) Validation of a Finite Element Approach (N3S) for Engine Applications, These Proceedings.

2 Halliday, J. (1999) Simulation of Engine Internal Flows using Digital Physics, These Proceedings.

3 Torres, A., and Henriot, S. (1996) Modeling the Effects of EGR Inhomogeneities Induced by Intake Systems in a FourValve Engine, SAE, No. 961959.

4 Zhu, Y.X., Savonen, C., Johnson, N., and Amsden, A. (1994) Three-Dimensional Computations of the Scavenging Process in an Opposed-Piston Engine, SAE, No. 941899.

5 Adamson, B., Gosman, A.D., Marooney, C.J., Nasseri, B., and Theodoropoulos, T. (1990) A New Unstructured-Mesh Method for Flow Prediction in Internal Combustion Engines, Proc. Comodia'90, Japan.

6 O’Rourke, P. (1999) A Parallel, Unstructured-Mesh Methodology for Device-Scale Combustion Calculations. These Proceedings.

7 Gosman, A.D. (1998) Developments in Industrial Computational Fluid Dynamics. Trans. IChemE., 76, Part A, 153-161.

8 Jasak, H., Weller, H.G., and Gosman, A.D. (1999) High Resolution NVD Differencing Scheme for ArbitrarilyUnstructured Meshes. Int. J. Numer. Meth. Fluids, to be published.

9 Issa, R.I., Ahmadi-Befrui, B., Beshay, K., and Gosman, A.D. (1991) Solution of the Implicitly Discretised Reacting Flow Equations by Operator-Splitting, J. Comp. Phys., 93, 388-410.

10 Muzaferija, S., and Gosman, A.D. (1997) Finite-Volume CFD Procedure and Adaptive Error Control Strategy for Grids of Arbitrary Topology. J. Comp. Phys., 138, 766-787.
11 Jasak, H., Luo, J.Y., Kaludercic, B., Gosman, A.D., Echtle, H., Liang, Z., Wirbeleit, F., Wierse, M., Rips, S., Werner, A., Fernström, G., and Karlsson, A. (1999) Rapid CFD Simulation of Internal Combustion Engines. SAE No.199901-1185.

12 Jones, P. (1999) Rapid Meshing Methodology for Internal Combustion Engines, Computational Dynamics Ltd..

13 Henriot, S. CFD-Aided Development of a SI-DI Engine. These Proceedings.

14 Wierse, A, Lang, U., and Ruhle, R. (1993) Architectures of Distributed Visualization Systems and their Enhancements. Eurographics Workshop on Visualization in Scientific Computing, Abingdon.

15 Launder, B.E., and Spalding, D.B. (1974) The Numerical Computation of Turbulent Flow. Comp. Meth. in Appl. Mech. \& Eng., 3, 269.

16 Ahmadi-Befrui, B., and Gosman, A.D. (1989) Assessment of Variants of k- $\varepsilon$ Turbulence Model for Engine Flow Applications. Paper 343, Int. J. Num. Methods in Fluids.

17 El Tahry, S.H. (1983) k-ع Equations for Compressible Reciprocating Engine Flows. AIAA J. Energy, 7, 4.

18 Yakhot, V., Orszag, S.A., Thangam, S., Gatski, T.B., and Speziale, C.G. (1992) Development of Turbulence Models for Shear Flows by a Double Expansion Technique. Phys. Fluids, A4, 7, 1510-1520.

19 Shih, T.H., Zhu, J., and Lumley, J.L. (1993) A Realizable Reynolds Stress Algebraic Equation Mode. NASA TM105993.

20 Lebrere, L., and Dillies, B. (1996) Engine Flow Calculations Using a Reynolds Stress Model in the Kiva-II code, SAE 960636

21 El Tahry, S., and Howarth, D.C. (1992) Directions in Turbulence Modeling for In-Cylinder Flows in Reciprocating Engines. AIAA J. Propulsion and Power, 8, 1992

22 Haworth, D. (1999) Large-Eddy Simulation of In-Cylinder Flows. These Proceedings.

23 Bo, T., Clerides, D., Gosman, A.D., and Theodossopoulos, P. (1997) Prediction of the Flow and Spray Processes in an Automobile DI Diesel Engine. SAE, No. 978802.

24 Angelberger, C., Poinsot, T., and Delhaye, B. (1997) Improving Near-Wall Combustion and Wall Heat Transfer Modeling in SI Engine Computations. SAE , No. 972881 .

25 Baritaud, T.A, Duclos, J.M., and Fusco, A. (1996) Modeling Turbulent Combustion and Pollutant Formation in Stratified Charge SI Engines. 26th Symp. (Int.) on Combustion, Naples, 28 July-2 August.

26 Zhao, F.Q., Ming-Chia, L., and Harrington, D.L. (1997) A Review of Mixture Preparation and Combustion Control Strategies for Spark-Ignited Direct-Injection Gasoline Engines. SAE, No. 970627, 1997.

27 Duclos, J.M., and Zolver, M. (1998) 3D Modeling of Intake, Injection and Combustion in a DI-SI Engine Under Homogeneous and Stratified Operating Conditions. Proc. Comodia'98, 333-340.

28 Reitz, R. (1999) Developments in Spray Modeling in Diesel and Direct Injection Gasoline Engines. These Proceedings.

29 Gosman, A.D., and Clerides, D. (1997) Diesel Spray Modeling: a Review. Proc. ILASS-Europe Annual Meeting, Florence.

30 Schmidt, D.P., Rutland, C.J., and Corradini, M.L. (1997) A Numerical Study of Cavitating Flow Through Various Nozzle Shapes. SAE, No. 971597. 
31 Von Dirke, M. (1999) Simulation of Cavitating Flow in Diesel Injectors. These Proceedings.

32 Huh, K.Y., and Gosman, A.D. (1991) A Phenomenological Model of Diesel Spray Atomisation. Proc. Int. Conf. on Multiphase Flows, Tsukuba .

33 Arcoumanis, C., Gavaises, M., Nouri, J.M., Abdul-Wahab, E., and Horrocks, R.W. (1998) Analysis of the Flow in the Nozzle of a Vertical Multi-Hole Diesel Engine Injector. SAE, No. 980811.

34 Naitoh, N. (1999) Cyto-Fluid Dynamic Theory of Atomisation Processes. These Proceedings.

35 Reitz, R.D. (1987) Modeling Atomisation Processes in HighPressure Vaporising Sprays. Atomisation and Spray Technology, 3.

36 Bai, C., and Gosman, A.D. (1995) Development of Methodology for Spray Impingement Simulation. SAE, No. 950283 .

37 Nagaoka, M., Kawazoe, H., and Nomura, N. (1994) Modeling Fuel Spray Impingement on a Hot Wall for Gasoline Engines. SAE, No. 9405254.

38 Bai, C., and Gosman, A.D. (1996) Mathematical Modeling of Wall Films Formed by Impinging Sprays. SAE, No. 960626.

39 Stanton, D.W., and Rutland, C.J. (1996) Modeling Fuel Film Formation and Wall Interaction in Diesel Engines. SAE, No. 960628.

40 Habchi, C., Foucart, H., and Baritaud, T. (1999) Influence of Wall Temperature on Mixture Preparation in DI Gasoline Engines. These Proceedings.

41 Mantzaras, J., Felton, P.G., and Bracco, F. (1989) Fractals and Turbulent Engine Flames. Combustion and Flame, 77.

42 Boudier, P., Henriot, S., Poinsot, T., and Baritaud, T., (1992) A Model for Turbulent Flame Ignition and Propagation in Spark Ignition Engines. 24th Symp. (Int.) on Combustion, Sydney, 5-10 July.

43 Weller, H.G., Uslu, S., Gosman, A.D., Maly, R.R., Herweg, R., and Heel, B. (1994) Prediction of Combustion in HomogeneousCharge Spark-Ignition Engines. Proc. Comodia'94, Yokohama.

44 Heel, B., Maly, R., Weller, H.G., and Gosman, A.D. (1998) Validation of SI Combustion Model Over Range of Speed Load, Equivalence Ratio and Spark Timing. Proc, Comodia'98, Kyoto.

45 Peters, N. (1992) A Spectral Closure for Premixed Turbulent Combustion in the Flamelet Regime. J. Fluid Mech., 242.

46 Poinsot, T.J., Haworth, D.C., and Bruneaux, G. (1993) Direct Numerical Simulation and Modeling of Flame Wall Interaction for Premixed Turbulent Combustion. Combustion and Flame, 95, 118-132.

47 Torres, A., and Henriot, S. (1994) 3D Modeling of Combustion in Lean Burn Four-Valve Engines: Influence of Intake Configuration. Proc. Comodia'94, 139.
48 Baritaud, T.A., Duclos, J.M., and Fusco, A. (1996) Modeling Turbulent Combustion and Pollutant Formation in Stratified Charge SI Engines. 26th Symp. (Int.) on Combustion, Naples, 28 July-2 August.

49 Tang, H., Weller, H., and Gosman, A.D. Validation of a Wrinkled Flame Model of Partially-Premixed Combustion, in preparation.

50 Dekena, M., and Peters, N. (1999) Combustion Modeling with the G-Equation. These Proceedings.

51 Moriyoshi, Y., and Morikawa, H. (1996) Combustion Enhancement of Very Lean Premixture Part in Stratified Charge Condition. SAE, No. 962087.

52 Gosman, A.D., Marooney, C.J., and Weller, H.G (1990) Prediction of Unburnt Gas Temp in Multi-Dimensional Engine Combustion Simulation. Proc. Comodia'90, Japan.

53 Heywood, J.B. (1988) Internal Combustion Engine Fundamentals, McGraw-Hill, New York.

54 Magnussen, B.F., and Hjertager, B.W. (1981) On the Structure of Turbulence and a Generalised Eddy Dissipation Concept for Chemical Reaction in Turbulent Flow, 19th AIAA Aerospace Meeting, St. Louis, USA.

55 Kong, S.C., Han, Z., and Reitz, R.D. (1978) The Development and Application of a Diesel Ignition and Combustion Model for Multi-Dimensional Engine Simulation. SAE 950278.

56 Patterson, M.A., Kong, S.G., Hampson, G.J., and Reitz, R.D. (1994) Modeling the Effects of Fuel Injection Characteristics on Diesel Engine Soot and $\mathrm{NO}_{\mathrm{x}}$ Emissions, SAE 940523.

57 Bo, T., Clerides, D., Gosman, A.D., and Theodossopoulos, P. (1997) Multi-Dimensional Modeling of Diesel Combustion and Emissions Formation.Proc. First Meeting of the Greek Section of the Combustion Institute, 283-292.

58 Zolver, M., Griard, C., and Henriot, S., (1997) 3D Modeling Applied to the Development of a DI Diesel Engine: Effect of Piston Bowl Shape. SAE 971599.

59 Dillies, B., Marx, K., Dec, J., and Espey, C. (1993) Diesel Engine Combustion Modeling Using the Coherent Flame Model, SAE 930074.

60 Barths, H., Pitsch, H., and Peters, N. (1999) ThreeDimensional Simulation of DI Diesel Combustion and Pollutant Formation Using a Two-Component Reference Fuel. These Proceedings.

61 Naitoh, K., Itoh, H., Takagi, Y., and Kuwahara, K. (1992) Large Eddy Simulation of Premixed Flames in IC Engines. SAE 920590.

62 Sheppherd, C. (1998) Private Communication.

63 Weller, H., Tabor, G., and Gosman, A.D. (1998) Application of a Flame-Wrinkling LES Combustion Model to a Turbulent Mixing Layer. Proc. 27th Combustion Symp.

Final manuscript received in March 1999 